\title{
Pseudotumor cerebri controlled with ventriculo-peritoneal shunt as a rare complication of ROHHAD syndrome: a case report
}

\author{
Abdullah Al Shamrani' ${ }^{1}$, Ashwaq Al Tawil2, Afaf A Bin-Khathlan ${ }^{3}$, Ali H. Alwadei ${ }^{4}$ \\ ${ }^{1}$ Prince Sultan Medical Military City (PSMMC), ${ }^{2}$ Pediatric Endocrine Division, King Fahad Medical City \\ (KFMC), ${ }^{3}$ Ophthalmology Department, (KFMC), \\ ${ }^{4}$ National Neuroscience Institute, (KFMC), Riyadh, Saudi Arabia, KSA
}

Corresponding author: Abdullah Al-Shamrani, Email: Shamrani999@ hotmail.com, Orcid.org/0000-0001-7157-1706, Mobile no. 00966553367555

\begin{abstract}
Background: rapid-onset obesity with hypoventilation, hypothalamic dysfunction and autonomic dysregulation (ROHHAD) is a rare yet possibly fatal condition. It is similar to common obesity syndromes (whether exogenous or genetic) sideways with other endocrinopathy in early childhood. Objective of the study: this study aimed to present the clinical manifestations, laboratory and imaging results as well as complications and treatment particularly for ventriculo-peritoneal shunt for a ROHHAD patient with pseudotumor cerebri. Patient and methods: in this case report, a 10-year-old girl was presented to the emergency department with recurrent episodes of dyspnea, hypernatremia and cyanosis. Results: her obesity was controlled with sleeve surgery and hormonal evaluation showed high prolactin level and hypothyroidism. She continued to have symptomatic hypoventilation and severe Pseudo-Tumor Cerebri (PTC), idiopathic intracranial hypertension (IIH), in addition to significant reduction in her visual acuity. She was diagnosed with ROHHAD syndrome based on the clinical, laboratory and radiological findings. Brain and abdominal magnetic resonance imaging were performed to demonstrate a possible accompanying neural crest tumor, which was normal, but followup images were recommended. Conclusion: ROHHAD syndrome should be suspected in all cases of rapid onset obesity associated with hypothalamic-pituitary endocrine dysfunction. Also provided the high prevalence of cardiorespiratory arrest and potentially associated neural crest tumors, early recognition of ROHHAD syndrome is quite crucial to avert mortality and morbidity. Moreover, Early ventriculoperitoneal shunt insertion might be a lifesaving procedure to avoid visual impairment and subsequent blindness in severe pseudotumor cerebri.
\end{abstract}

Keywords: obesity, hypoventilation, hypothalamus, papilledema, hypernatremia, shunt, pseudotumor cerebri, idiopathic intracranial hypertension.

\section{INTRODUCTION}

In 1965, the first case of rapid-onset obesity with hypothalamic dysfunction, hypoventilation, and autonomic dysregulation (ROHHAD) was reported ${ }^{[1]}$. ROHHAD is defined as a sporadic disorder with abnormalities including significant weight gain, breathing problem (hypoventilation) and other disorders in both endocrine and autonomic nervous system ${ }^{[2]}$. Ize-Ludlow et al. reported fifteen patients with the condition [3]. ROHHAD syndrome is potentially lethal and the reason behind the high morbidity and mortality rate was the high incidence of cardiorespiratory arrest which could be prevented by the early recognition of the breathing disorder as well as early ventilatory support [4-6]. The criteria for diagnosis of ROHHAD can be summarized as follows: 1) rapid-onset obesity 2) alveolar hypoventilation starting after the age of 1.5 years, 3) evidence of hypothalamic dysfunction, as defined by 1 or more of the following findings: 
hyperprolactinemia, disordered water balance (hypodipsia), central hypothyroidism, failed growth hormone stimulation test, corticotrophin deficiency, or altered onset of puberty (delayed or precocious), and 4) absence of PHOX2B mutation [7]. In differential diagnosis, negative PHOX2B sequencing results indicated that this entity was distinct from congenital central hypoventilation syndrome (CCHS) ${ }^{[8]}$. ROHHAD may have different clinical presentations. Moreover, being rare, it can be easily missed, and since the disease was progressive this could lead to serious complications and potential deaths ${ }^{[4]}$.

\section{CASE REPORT}

A 10-year-old girl presented to our emergency department with an acute history of respiratory distress, cyanosis and loss of consciousness. Since the age of 3 years, she had experienced rapid increase in her weight and on further interview, she had hypodipsia. Two years, she presented with severe respiratory distress and cyanosis and required prolonged ventilation in the pediatric intensive care unit with difficult extubation due to her poor respiratory effort. A thyroid function test done at that time and showed central hypothyroidism, and 25 micrograms of thyroxin was started. MRI scans of the brain and adrenals were performed and were normal. A sleeve gastrectomy was performed 4 months ago for her morbid obesity. She had a BMI of 46 .

Following discharge, she was dependent on bilevel positive airway pressure (BiPAP) at home. During this admission, she had respiratory acidosis with $\mathrm{CO}_{2}$ retention and required observation in the PICU for three nights until she stabilized and continued BiPAP while sleeping in the pediatric ward. By physical examination she looked unwell with apparent respiratory distress. She had obesity with a weight of $93 \mathrm{~kg}$, height of $135 \mathrm{~cm}$ and a BMI of 46. Her Tanner stage was 3. The remaining physical exam was unremarkable, and she had normal muscle power and neurological function. The laboratory workup showed high sodium of $165 \mathrm{mmol} / \mathrm{L}$ (normal 135-145) corrected with isotonic solutions. Serum osmolality was high at $329 \mathrm{mosmol} / \mathrm{L}$ (normal 275-295) and urine osmolality was normal at $853 \mathrm{mosmol} / \mathrm{L}$, which excluded diabetes insipidus. The hormonal workup showed high prolactin at $793 \mathrm{mlU} / \mathrm{L}$ (normal 102-496) with normal follicular stimulating hormone, luteinizing hormone and estrogen levels. The PHOX2B gene was negative.

The thyroid function test was repeated as the patient was symptomatic with lethargy and cold intolerance and had a T4 of $12.9 \mathrm{pmol} / \mathrm{L}$ (normal 12-22) and TSH of $2.390 \mathrm{mIU} / \mathrm{l}$ (normal 0.28-4.3). Her thyroxine dose was increased to 37.5 micrograms. Due to her previous surgery, her vitamin levels were checked and were normal. She complained of persistent headache associated with double vision, which is a red flag for pseudotumor cerebri. We therefore excluded potential secondary causes for intracranial hypertension with CT scan and later MRI with MRV to rule out cerebral venous sinus thrombosis. Ophthalmology confirmed optic nerve involvement with reduction in visual acuity to 20/40 in the right eye and 20/60 in the left eye along with sluggish pupil reaction on both sides with no afferent pupillary defect. Her visual field showed an enlarged blind spot and gross reduction in sensitivity to light on both sides (Fig.1). 

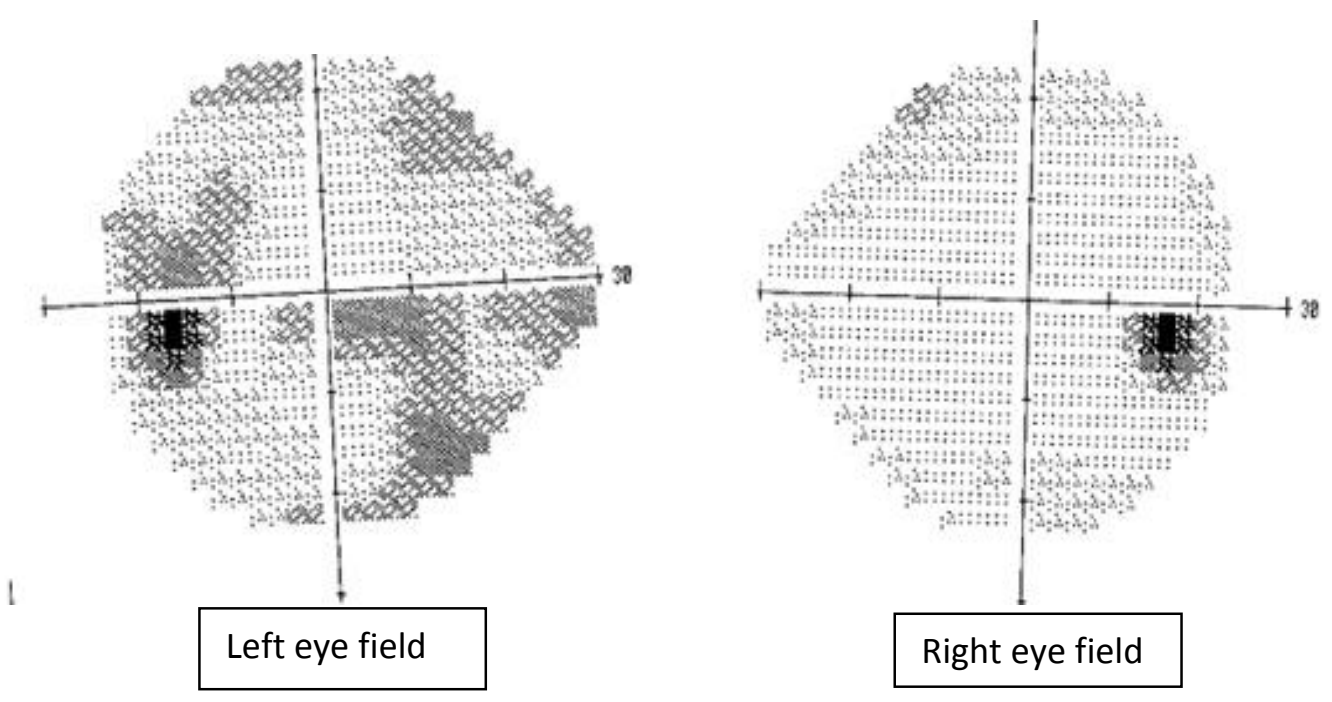

Figure 1: visual field showing an enlarged blind spot and gross reduction in sensitivity to light on both sides

Examination of the optic nerve showed elevation of the optic nerve with diffused pallor and this indicated chronicity of the papilledema with ultrasound and we found a thickness of the optic nerve of $5.7 \mathrm{~mm}$ on the right and $5.2 \mathrm{~mm}$ on the left. Lumbar puncture was performed and $40 \mathrm{ml}$ of cerebrospinal fluid was drained with an opening pressure of more than $54 \mathrm{~cm}$ $\mathrm{H}_{2} \mathrm{O}\left(540 \mathrm{mmH}_{2} \mathrm{O}\right)$ and a closing pressure of 18 $\mathrm{cm} \mathrm{H}_{2} \mathrm{O}$ measured and drained by our interventional neuroradiologist under fluoroscopy guidance, as the patient was morbidly obese. This procedure was done without bedside trials, as we knew LP was technically impossible in her condition. A repeated lumbar puncture was done due to persistent headache with an opening pressure of more than $52 \mathrm{~cm} \mathrm{H}_{2} \mathrm{O}$. She was administered acetazolamide, but had a significant side effect of acidosis such that a ventriculoperitoneal shunt was inserted as advised by the neurology team. The patient's clinical condition was improved with restoration of her vision and the headaches resolved. She showed some aggressive and oppositional behavior that needed input from the behavioral and psychology team. We were unable to run her polysomnography, as we lacked a sleep technician at that time. She was discharged home on BiPAP at night and will be seen in multiple clinics to follow her clinical condition. Of note, her blood pressure was within normal limits, which excluded a condition that mimics
IIH or malignant hypertension. As she underwent sleeve surgery and was on thyroxine for hypothyroidism, we excluded all common secondary metabolic and vitamin deficiencies as the cause of IIH.

\section{DISCUSSION}

In 2009, researchers noticed a mutation gene that triggers CCHS. Recognition and treatment of this disease were crucial to avoid the high prevalence of cardiorespiratory arrest and serious complications ${ }^{[9]}$. The diagnosis of ROHHAD can be raised if rapid (within 6 to 12 months) and dramatic (often over 20 pounds) weight gain was noticed in the presence of abnormal increase in desire to eat. This rapidonset obesity is considered a warning signal of hypothalamic dysfunction ${ }^{[10]}$. Moreover, other hypothalamic abnormalities might appear at any time from months to years succeeding the rapid-onset obesity. Common abnormalities such as high prolactin levels, inability to maintain normal water balance in the body (resulting in abnormally high or low sodium levels, low thyroid hormone, low cortisol and growth hormone deficiency and early or late puberty might also arise [11, 12]. Alveolar hypoventilation and inability to increase breathing in response to abnormal oxygen or carbon dioxide levels, is constant feature of ROHHAD and it is the most dangerous feature, pulsatile tinnitus, is common in IIH and, with accompanying headaches, is somewhat specific for the diagnosis [13]. 
However, our patient did not display this symptom.Treatment for IIH had two major objectives: 1) alleviation of symptoms (usually headache) and 2) preservation of vision. Usually, a multidisciplinary approach is needed in the management of IIH with involvement of an ophthalmologist, neurologist, neurosurgeon, interventional neuro-radiologist and nutritionist (for weight loss). Treatment options included medications, serial LPs and surgery. Medications included loop diuretics, such as furosemide (Lasix), carbonic anhydrase inhibitors (acetazolamide and Topamax), corticosteroids and indomethacin. Few surgical options are available, including shunting with either a ventriculoperitoneal shunt (VPS) or lumboperitoneal shunt (LPS), optic nerve sheath fenestration, and venous stenting. Acetazolamide is the typical first line treatment for IIH ${ }^{[14]}$. Indomethacin is effective in the treatment of secondary intracranial hypertension [15]. Despite the multiple options for medications, we elected to insert a VPS within a couple of weeks of establishing the diagnosis, as medical therapy was not an option. The patient did not tolerate acetazolamide with severe acidosis after in initial small single dose of $125 \mathrm{mg}$. This reaction was confirmed by repeating the dose a few times. Topamax would have been an excellent choice in this case given her obesity, but it usually takes weeks to months to produce an effect and has the same risk of inducing acidosis. Corticosteroids were also not considered because of the major side effects and obesity. Serial LPs were not an option for many reasons, including CSF reforms within six hours unless there is a CSF leak, making any treatment benefit only last a short time. Lumbar punctures are uncomfortable for most patients and painful for many and have their own complications (e.g., low pressure headaches, CSF leak, CSF infection, intraspinal epidermoid tumors). Lumbar punctures are often technically difficult in obese patients [16]. A relatively new and somewhat controversial treatment option for $\mathrm{IIH}$ as venous stenting was discussed and not used in our patient ${ }^{[14,17]}$. The decision to insert a VPS was made after careful discussion with the family and health care professionals, keeping in mind the seriousness of permanent vision loss as a major morbidity associated with IIH. An early, hospital-based study that followed 57 patients for 5 to 41 years found that 24 percent developed blindness or severe visual impairment [18, 19] Patients required regular follow-up visits until they were stabilized. Follow-up visit intervals were individualized based on the severity, duration and response to treatment of the clinical manifestations, but initially should be at least monthly. Each office visit should include a best corrected visual acuity, formal visual field testing, and dilated fundus examination with optic disc photographs [20]. Our patient was controlled with BiPAP at $22 / 10$ and $25 \%$ oxygen with a pack up rate of $24 / \mathrm{m}$. usually, patients will progress gradually, and symptoms of autonomic dysregulation by a median age of 3.6 years was started to be apparent, which included altered sweating and gastrointestinal dysmotility. Our patient continued to have diarrhea with dehydration and hypernatremia, ophthalmic manifestations, thermal dysregulation and altered pain perception [3]. Luckily, our patient was negative for anatomic malformations of the autonomic nervous system such as ganglioneuromas or ganglioneuroblastomas. Other features in a subset of individuals with ROHHAD include behavioral disorders, developmental disorders with very low intelligence quotient (IQ) ${ }^{[11]}$, which was obvious in our case due to her aggression toward her mom and nurses looking after her and oppositional behavior toward instructions given by the treating team. There were no seizures reported, which could be related to low oxygen levels. The uniqueness of this case was the severity of her headaches, which were correlated to $\mathrm{CO}_{2}$ retention and confirmed to have severe papilledema and marked reduction in her visual acuity. Her optic nerve was thicker than $5.2 \mathrm{~mm}$, which has a normal cutoff of 11 , but her blood pressure and heart rate remained in the normal range. However, it may take a several years after the onset of rapid weight gain to start having other symptoms of hypothalamic dysfunction, autonomic dysregulation and/or hypoventilation. This could be one reason for missing or delay in reaching the appropriate 
diagnosis, long-term follow-up with a high level of suspicion is needed ${ }^{[12]}$. Once the diagnosis is suspected, comprehensive respiratory assessment is needed with possible need for BiPAP titration to eliminate hypoventilation during wakefulness and sleep [4]. The sleeve is a lifesaving treatment for morbid obesity, especially in patients with exceeding BMI of 46 and our patient lost $17 \mathrm{~kg}$ over a 4-month period with no apparent complications despite being too early to judge. It is important to follow the patient with repeated polysomnography every 3-6 months to ensure optimal oxygenation and ventilation. A scheduled screening of neural crest tumors should be performed every 1-2 years by chest and abdominal imaging [3]. It's important to mention that control of breathing deficits is one of the main challenges in ROHHAD, the goal in such cases is optimization the oxygenation and ventilation ${ }^{[9]}$. At some point, in some children artificial ventilation might be required with ROHHAD during sleep, though signs of hypoventilation may not occur immediately after onset of rapid weight gain.

\section{CONCLUSION}

Multidisciplinary care is crucial to the successful management of ROHHAD patients and the affected individuals will likely suffer from neurocognitive, blindness, compromise and sudden death if inadequately treated,. Hypoventilation is often usually controlled with BiPAP. A high index of suspension is recommended for monitoring autonomic dysfunction and signs and symptoms of increased intracranial pressure. Early insertion of a VP shunt can preserve vision and is a simple and noninvasive method for patients with controlled pseudotumor cerebri who fail or cannot tolerate medical therapy.

\section{Consent}

Written informed consent was obtained from the patient's legal guardian(s) for publication of this case report and any accompanying images. In addition, our local ethics committee (IRB) of the King Fahad Medical City (KFMC) approved the research.

\section{Competing interests}

The authors declare that they have no competing interests.
Availability of data and materials: data sharing wass not applicable to this article, as no datasets were generated or analyzed for the current study.

\section{Abbreviations:}

ROHHAD: Rapid-onset obesity with hypothalamic dysfunction, hypoventilation, and autonomic dysregulation, PTC: Severe pseudo tumor cerebri, IIH: Idiopathic Intracranial Hypertension, CCHS: congenital central hypoventilation syndrome

VPS: Ventriculoperitoneal Shunt, LPS: Lumboperitoneal Shunt, $\mathbf{H}_{2} \mathrm{O}$ : Water, CSF: Cerebrospinal fluid, TSH: thyroid stimulating hormone. BiPAP: Bi level positive airway pressure.

\section{REFERENCES}

1. Fishman LS, Samson JH and Sperling DR (1965): Primary alveolar hypoventilation syndrome (Ondines's Curse) Am. J. Dis. Child., 110:155-1661.

2. Proulx F, Weber ML, Collu R, Lelievre M, Larbrisseau A and Delisle $M$ (1993): Hypothalamic dysfunction in a child: a distinct syndrome? Report of a case and review of the literature. Eur. J. Pediatr., 152 (6):526-535.

3. Ize-Ludlow D et al. (2007): Rapid-onset obesity with hypothalamic dysfunction, hypoventilation, and autonomic dysregulation presenting in childhood. Pediatrics, 120 (1): 79-88.

4. Al-Harbi AS, Al-Shamrani A and AlShawwa BA (2016): Rapid-onset obesity, hypothalamic dysfunction, hypoventilation, and autonomic dysregulation in Saudi Arabia.Saudi Med. J., 37(11):1258-1263.

5. Bougneres $\mathbf{P}$, Pantalone $\mathbf{L}$, Linglart $A$, Rothenbuhler A and Le Stunff C (2008): Endocrine manifestations of the rapid-onset obesity with hypoventilation, hypothalamic, autonomic dysregulation, and neural tumor syndrome in childhood. J. Clin. Endocrinol. Metabol., 93(10):3971-3982.

6. Gothi D and Joshi JM (2005): Late onset hypoventilation syndrome: is there a spectrum of idiopathic hypoventilation syndromes? Indian J. Chest Dis. Allied Sci., 47(4):293-300.

7. Amiel J, Laudier B, Attie-Bitach T, Trang H, de Pontual $\mathrm{L}$ et al. (2003): polyalanine 
expansion and frameshift mutations of the paired- like homebox gene PHOX2B in congenital central hypoventilation. Nature Genetics, 33: 459-461.

8. Gallego J and Dauger S (2008): PHOX2B mutations and ventilator control. Respir. Physiol. Neurobiol., 164: 49-54.

9. Weese-Mayer DE, Berry-Kravis EM, Ceccherini I, Keens TG, Loghmanee DA and Trang H (2010): Congenital central hypoventilation syndrome: genetic basis, diagnosis and management. Am. J. Respir. Crit. Care Med., 181:626-644

10. Durivage SK, Winter RJ, Brouillette RT, Hunt CE and Noah Z (1985): Idiopathic hypothalamic dysfunction and impaired control of breathing. Pediatrics, 75(5):896-904.

11. Patwari PP, Zelko FA et al. (2010): Rapidonset obesity with hypothalamic dysfunction, hypoventilation, and autonomic dysregulation (ROHHAD. Am. J. Respir. Crit. Care Med., 181: 2435-2442.

12. Rand CM et al. (2011): Rapid-onset obesity with hypothalamic dysfunction, hypoventilation and autonomic dysregulation: analysis of hypothalamic and Autonomic candidate genes. Pediatr. Res., 70(4): 375-383.

13. Giuseffi V, Wall M, Siegel PZ and Rojas PB (1991): Symptoms and disease associations in idiopathic intracranial hypertension (pseudo tumor cerebri): a case-control study. Neurology, 41:239-243.
14. Biousse V, Bruce BB and Newman NJ (2012): Update on the pathophysiology and management of idiopathic intracranial hypertension. J. Neurol. Neurosurg. Psychiatry, 83:488-592.

15. Rasmussen $M$ (2005): Treatment of elevated intracranial pressure with indomethacin: friend or foe? Acta Anaesthesiol. Scand., 49:341-353.

16. Corbett JJ and Mehta MP (1983): Cerebrospinal fluid pressure in normal obese subjects and patients with pseudotumor cerebri. Neurology, 33:13861395.

17. Friedman DI (2006): Cerebral venous pressure, intra-abdominal pressure, and dural venous sinus stenting in idiopathic intracranial hypertension. J. Neuroophthalmol., 26:61-74.

18. Corbett JJ, Savino PJ, Thompson HS et al. (1982): Visual loss in pseudotumor cerebri. Follow-up of 57 patients from five to 41 years and a profile of 14 patients with permanent severe visual loss. Arch Neurol., 39:461-473.

19. Moretti R, Pizzi B, Cassini F and Vivaldi N (2009): Reliability of optic nerve ultrasound for the evaluation of patients with spontaneous intracranial hemorrhage. Neurocrit. Care. Dec., 11(3):406-410.

20. Wall M (1990): Sensory visual testing in idiopathic intracranial hypertension: measures sensitive to change. Neurology, 40:1859-1864. 